\title{
Neurocognitive and psychiatric changes as the initial presentation of neurosyphilis
}

\author{
Cecilia T. Costiniuk MD, Paul A. MacPherson PhD MD
}

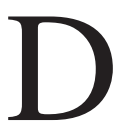
espite its recognition for centuries as "the great imitator," neurosyphilis remains a diagnostic challenge because of its wide spectrum of presentations. Here we describe 3 men with neurosyphilis in whom diagnosis and treatment were delayed for up to a year because their initial presentations were in keeping with primary neurological or psychiatric problems.

\section{Cases}

\section{Case 1}

A 40-year-old heterosexual man was admitted to a psychiatric ward with a 2-3 week history of excessive talking with senseless conversation, excessive activity, decreased sleep and delusions of grandiosity. The patient had been well previously other than having depression more than 10 years earlier and chronic back pain. Bipolar disorder with manic episode was diagnosed.



Figure 1: Magnetic resonance imaging scan of the brain in case 1, showing diffuse, increased mineralization (arrows) in the basal ganglia, with bilateral involvement of the caudate nucleus and putamen.
Investigations included magnetic resonance imaging (MRI), which showed mineral deposits within the globus pallidus (Figure 1); however, screening for Wilson disease was negative. Further work-up included measurement of iron, ferritin, thyroid-stimulating hormone, vitamin $B_{12}$ and folate levels, all of which were within normal limits. Results of toxicology and serologic HIV testing were also negative.

Upon admission, the patient received treatment with lithium and olanzapine, as well as temporary sedation with clonazepam. Over the next few months, a mask-like facies developed, his speech became slow and stuttered, and a tremor developed in his right hand. The patient had reduced strength, and his deep tendon reflexes were brisk on the right side. His gate was shuffling, and he had difficulty with turning.

The development of neurologic symptoms prompted serologic testing for syphilis. Enzyme immunoassay was positive for antibodies to Treponema pallidum, the T. pallidum particle agglutination assay was reactive, and the rapid plasma reagin test was reactive with a titre of 1:128. Subsequent analysis of the cerebrospinal fluid (CSF) showed a leukocyte count of 42 (normal $<5) \times 10^{6} / \mathrm{L}$ with $91 \%$ lymphocytes, a protein level of 1.43 (normal 0.15-0.45) g/L and a Venereal Disease Research Laboratory (VDRL) titre of 1:4 with a reactive $T$. pallidum particle agglutination assay. The patient indicated having 2 previous female sexual partners, and there was
Competing interests: None declared.

This article has been peer reviewed.

\section{Correspondence to: \\ Paul A. MacPherson, pmacpherson}

@ottawahospital.on.ca

CMAJ 2013. DOI:10.1503

/cmaj.121146

\section{- Ker POINTS}

- Neurosyphilis has a wide spectrum of neurocognitive symptoms that are nonspecific and common to many neurologic and psychiatric disorders.

- Given the resurgence of syphilis in the past decade, clinicians should consider serologic testing for the disease if patients present with neurocognitive changes.

- In patients with neurocognitive changes and positive results of serologic testing for syphilis, cerebrospinal fluid should be analyzed for lymphocytic pleocytosis, an elevated protein level and a reactive Venereal Disease Research Laboratory test. 
no prior diagnosis of syphilis or previous symptoms to suggest primary or secondary syphilis.

After referral to our clinic, the patient received penicillin $G$ intravenously for 2 weeks. His condition improved noticeably, although he was left with some residual cognitive and physical deficits. The serum rapid plasma reagin titre was 1:16 at follow-up 6 months after treatment. Repeat lumbar puncture at that time showed a substantial decline in the leukocyte count and protein level, and the VDRL converted to nonreactive. Two years after treatment, his serum rapid plasma reagin titre declined to 1:8.

At the most recent follow-up, 4 years after neurologic treatment was stopped, the patient still had dysarthria with some echolalia and exhibited akathisia. He still had bradykinesia with some difficulty in mobility, a fine tremor in his right hand and hyperreflexia on the right side. He also reported recurrent sharp pains shooting down his left leg. Before his initial presentation, he was independent and worked in a plant as a welder in a supervisory role. Currently, he lives in a group home, and although he can manage his basic activities of daily living, he is unable to do any of his instrumental activities of daily living.

\section{Case 2}

A 57-year-old heterosexual man presented with new-onset neurologic symptoms. These included 2 focal seizures on the right side with generalization, followed by a postictal phase. Other changes included decreased cognitive function and impairment in short-term memory, which had developed over the preceding year, as well as decreased visual acuity bilaterally. His medical history included chronic alcohol use and cardiovascular disease with a myocardial infarction 10 years previously and evidence of a previous stroke on MRI scans.

Findings on neurologic examination were unremarkable, although ophthalmologic examination revealed bilateral temporal pallor with acuity of 6/60 and 6/180 in the right and left eyes, respectively.

An MRI scan showed evidence of previous ischemia subcortically with wallerian degeneration in the midbrain and a small cortical focus. An electroencephalogram showed intermittent slowing of cerebral activity over the frontotemporal regions, especially in the left hemisphere. No obvious epileptiform activity was observed. Results of routine blood work and metabolic screening were within normal limits. Treatment with phenytoin was started following the seizures. The patient continued to carry out his activities of daily living but was unable to work.
Ten months after his initial presentation, serologic testing for syphilis was requested because the patient's findings could not confidently be attributed to his cardiovascular disease or chronic alcohol use. Chemiluminescent microparticle immunoassay and T. pallidum particle agglutination assay were reactive, as was the rapid plasma reagin test (titre 1:32).

Upon referral to our clinic, CSF analysis revealed lymphocytic pleocytosis (leukocyte count $93 \times 10^{6} / \mathrm{L}, 97 \%$ lymphocytes), a protein level of $0.79 \mathrm{~g} / \mathrm{L}$ and a VDRL titre of 1:4 with a reactive $T$. pallidum particle agglutination assay. The patient indicated having 10-15 female sexual partners in his lifetime. He had been in a monogamous relationship with his current partner for the past 15 years. The patient was given intravenous penicillin $\mathrm{G}$ treatment for 2 weeks. At follow-up 6 months after treatment, his condition was stable, with no further seizures since the initial presentation. There was no further deterioration in his memory or his vision, although neither had improved. Results of serologic tests revealed a rapid plasma reagin titre of 1:8.

\section{Case 3}

A 44-year-old homosexual man was assessed for a 1-year history of neurologic and psychiatric symptoms. His medical history included anxiety and depression and a suicide attempt more than 10 years earlier. His neurologic symptoms began the previous year with weakness in his left foot and leg that progressed to numbness, as well as a tremor in his left hand. Over the next few months, he began to experience dysarthria and stuttering of speech, difficulties in concentration, short-term memory loss and an inability to perform routine activities. As a result of these symptoms, he had to quit his job as a computer programmer.

Neuropsychological testing revealed cognitive deficits primarily in information processing. Findings on MRI of the brain were normal. Results of routine blood work and metabolic screening were within normal limits, and serologic HIV test results were negative. The patient's cognitive symptoms were thought to be related to anxiety, and treatment with citalopram and clonazepam was started.

Eleven months after presentation, the patient saw a new physician, who requested serologic testing for syphilis. Chemiluminescent microparticle immunoassay and T. pallidum particle agglutination assay were reactive, and the rapid plasma reagin titre was 1:32. Upon referral to our clinic, the patient was found to have dysarthria. Physical examination revealed no cranial nerve palsies or pupillary abnormalities. Power was decreased on flexion of the right hip and knee and 
on extension of both knees. The patellar reflex was reduced, and the ankle jerk was absent on the left side. Vibration and proprioception were decreased in both feet, and there was a fine intention tremor in both hands. Finger-to-nose movement was coarse because of the tremor. The patient's gait was unsteady but was neither broadbased nor shuffling. Analysis of CSF showed a leukocyte count of $144 \times 10^{6} / \mathrm{L}(95 \%$ lymphocytes), a protein level of $1.32 \mathrm{~g} / \mathrm{L}$ and a VDRL titre of 1:32 with a reactive T. pallidum particle agglutination assay. The patient described having several male sexual partners in his lifetime.

Penicillin $G$ was given intravenously for 2 weeks. By the end of treatment, the patient's speech was improved and was more fluid. At the most recent follow-up several years later, the rapid plasma reagin titre was $1: 8$. Although the numbness in his left leg had lingered for several months after treatment, it had since resolved. His cognitive symptoms had also resolved, and he had regained his baseline level of functioning.

\section{Discussion}

Canada has experienced a resurgence of syphilis in the past decade, with rates of 3.5 to 4.0 per 100000 in 2008. ${ }^{1}$ Outbreaks of infectious syphilis, especially among men who have sex with men, have been reported in all major Canadian cities as well as worldwide. ${ }^{1}$ The incidence of neurosyphilis in Canada, however, is unknown.

\section{Clinical presentation}

Neurosyphilis occurs in about $30 \%$ of people with untreated syphilis and can occur at any stage. ${ }^{2}$ The incubation period can be from less than 2 years to 20 years. ${ }^{1}$ Early neurosyphilis may be asymptomatic, or patients may present with syphilitic meningitis or syphilitic meningovasculitis. ${ }^{3}$ The former is associated with headache, whereas patients with syphilitic meningovasculitis may present with stroke, which typically involves the distribution of the middle cerebral artery. In late neurosyphilis, paralytic dementia, formerly known as general paresis of the insane, is characterized by progressive dementia with personality changes. ${ }^{3}$ Tabes dorsalis is another presentation seen in late neurosyphilis, whereby involvement of the posterior columns of the spinal cord results in sensory ataxia with bowel and bladder dysfunction. Space-occupying lesions contiguous with the dura may result from the formation of gummas. ${ }^{4}$ Argyll Robertson pupil and optic atrophy may also be observed in late neurosyphilis. ${ }^{3}$

In a retrospective study, Mitsonis and colleagues examined the clinical features of 81 patients presenting with neurosyphilis between 1965-84 and 1985-2005. ${ }^{5}$ They examined the prevalence of typical features, such as tabes dorsalis, progressive general paresis and meningovascular syphilis. Features of the classic presentations were much less frequent in the latter period than in the earlier period, a difference attributed to the widespread use of antibiotics and better screening practices. ${ }^{5}$ In all 3 cases we have described, the patients did not have pupillary abnormalities, and findings on cardiovascular and dermatologic examinations were unremarkable.

Table 1 describes the signs and symptoms of neurosyphilis and their frequencies. ${ }^{5-18} \mathrm{HIV}$-positive individuals are more likely than HIV-negative people to present with neurosyphilis, ${ }^{19}$ although the presenting symptoms are generally similar. ${ }^{18,19}$

In our case reports, the first patient's presentation was similar to that of other published cases of neurosyphilis whereby psychiatric manifestations resulted in hospital admission because of bizarre or violent behaviour. In the retrospective review by Mitsonis and colleagues, mental disorders occurred in $86 \%$ and cognitive impairment in $64 \%$ of patients presenting between 1985 and $2005 .{ }^{5}$ Our first case also highlights various other features in neurosyphilis, such as apparent mineral deposition in the basal ganglia observed on

Table 1: Frequency of signs and symptoms of neurosyphilis in people without HIV infection*

\begin{tabular}{|c|c|}
\hline Sign or symptom & Frequency, \% \\
\hline Asymptomatic ${ }^{5,6}$ & $\leq 37$ \\
\hline $\begin{array}{l}\text { Personality changes, depression, dementia or cognitive } \\
\text { changes, mania, paranoia }\end{array}$ & $33-86$ \\
\hline $\begin{array}{l}\text { Pupillary changes (Argyll Robertson pupils, anisocoria) } \\
\text { or ophthalmic symptoms (most commonly uveitis, } \\
\text { but also optic atrophy, blurred vision, reduced colour } \\
\text { perception) }\end{array}$ & $\leq 43$ \\
\hline Hearing impairment, cochleovestibular dysfunction ${ }^{7,10}$ & $3-10$ \\
\hline $\begin{array}{l}\text { Sensory impairment (decreased proprioception, } \\
\text { Romberg sign, reduced vibration sense, ataxia) }\end{array}$ & 24-48 \\
\hline Painful polyradiculopathy, lightning pains ${ }^{11}$ & Rare \\
\hline Myelopathy ${ }^{12}$ & 9 \\
\hline Parkinsonism, movement disorders ${ }^{13,14}$ & Rare \\
\hline Headache ${ }^{5,10}$ & $1-25$ \\
\hline Hyporeflexia, hypotonia ${ }^{7}$ & $10-50$ \\
\hline Stroke $e^{7,15}$ & $7-24$ \\
\hline Seizures ${ }^{5,15}$ & $1-25$ \\
\hline Bladder dysfunction $^{16}$ & Rare \\
\hline Gastric or visceral crises ${ }^{17}$ & Rare \\
\hline \multicolumn{2}{|c|}{$\begin{array}{l}\text { Note: "Tabes dorsalis" typically refers to a constellation of signs and symptoms (including } \\
\text { lightning pains, loss of peripheral reflexes, impaired vibration and position senses, ataxia, } \\
\text { urinary incontinence, impotence, optic atrophy and Argyll Robertson pupils), although signs } \\
\text { and symptoms are presented separately in this table. } \\
\text { *For information on neurosyphilis in HIV-positive individuals, see references } 10,18 \text { and } 19 .\end{array}$} \\
\hline
\end{tabular}


MRI scans. We found only one other reported case of a patient with neurosyphilis who had mineral deposition, presumed to be ferritin, in the basal ganglia. ${ }^{20}$ Whether mineral deposition in our patient's brain was related to syphilis, was an incidental finding or was due to a second concurrent disorder is unknown. Mineral deposition in the brain has previously been reported in patients with Parkinson disease and other neurodegenerative diseases..$^{20}$ Although there have been a few reported cases of neurosyphilis manifesting with parkinsonism, the incidence is thought to have declined in recent years and remains rare. ${ }^{13}$ Because the parkinsonian features in our first patient did not abate or reverse after treatment with penicillin and persisted unchanged over the 4 years after all neuroleptics were stopped, the relative contribution of neurosyphilis and neuroleptics to his parkinsonian features remains unclear. The patient's lancinating neuropathic leg pains, albeit rare, have been previously documented in cases of neurosyphilis. ${ }^{21}$ Winston and colleagues described the first case of early syphilis presenting as a polyradiculopathy before the development of the other clinical signs of syphilis. ${ }^{21}$

The second patient's history of past alcohol abuse made nutritional deficiency or toxic optic neuropathy plausible causes of his decreased visual acuity, and even now, these cannot be excluded as contributing factors. Furthermore, although the findings on his MRI scans were not consistent with Korsakoff syndrome, the patient's decline in short-term memory and wide-based gait could have been attributed to his alcohol use. Similarly, his history of hypertension and dyslipidemia supported the role of cardiovascular disease as the cause of the stroke documented on imaging. Meningovascular neurosyphilis can also result in ischemic stroke ${ }^{5}$ thus, we cannot determine with certainty whether his stroke was due to his cardiovascular risk factors or to the neurosyphilis.

This patient also experienced seizures, which may occur at different stages of neurosyphilis. Various types of seizures, including status epilepticus ${ }^{22}$ have been documented as the presenting feature in neurosyphilis in $3.5 \%{ }^{3}$ to $25 \%$ of patients. ${ }^{15}$ Involvement of almost any part of the eye is possible in neurosyphilis, and optic atrophy resulting in reduced visual acuity, as observed in this patient, has been previously described. ${ }^{23}$

As a homosexual man, the third patient is a member of a population at increased risk of syphilis. The diagnosis of neurosyphilis may have been initially overlooked because of his history of anxiety. Like the first patient, he exhibited some parkinsonian-like features. However, his cognitive symptoms completely resolved after treatment, and he regained his baseline level of functioning.

\section{Diagnosis}

A diagnosis of neurosyphilis is confirmed on the basis of a reactive serologic test for syphilis in CSF. Probable neurosyphilis involves a serologic diagnosis of syphilis of any stage; a negative VDRL test result in CSF; and the presence of an increased protein level or leukocyte count in CSF in the absence of another cause, plus clinical signs or symptoms consistent with neurosyphilis without any other clear cause of these abnormalities. ${ }^{1}$

\section{Treatment}

Optimal management of neurosyphilis consists of the administration of 3-4 million units of aqueous penicillin $\mathrm{G}$ intravenously every 4 hours for $10-14$ days. ${ }^{1}$ Penicillin desensitization is recommended for patients with a penicillin allergy. ${ }^{1}$ After the completion of treatment, repeat lumbar puncture at 6-12 months is needed to check whether CSF levels have returned to normal. ${ }^{1}$

Treatment requires obtaining appropriate levels of penicillin in the CSF to exert an antitreponemicidal effect. ${ }^{24}$ Because there have been reports of treatment failures despite appropriate penicillin therapy in primary syphilis, ${ }^{2,25}$ it is essential to ensure sufficient declines in titres and normal CSF levels following treatment. Data are limited to guide prognostication. In general, earlier treatment, to limit structural or tissue damage, may increase the probability of returning to one's neurocognitive baseline.

\section{Conclusion}

Taken together, the 3 patients we have described presented with a wide variety of nonspecific neurocognitive changes common to many neuropsychiatric disorders. These cases illustrate the challenge in diagnosing neurosyphilis and the need to consider neurosyphilis as a possible cause of neurocognitive abnormalities. Early testing for syphilis would permit appropriate, timely treatment and may optimize chances for recovery.

\section{References}

1. Public Health Agency of Canada. Canadian guidelines on sexually transmitted infections (revised January 2010). Ottawa (ON): The Agency; 2011. Available: www.phac-aspc.gc.ca/std-mts/sti-its (accessed 2012 Oct. 14).

2. Rolfs RT, Joesoef MR, Hendershot EF, et al. A randomized trial of enhanced therapy for early syphilis in patients with and without human immunodeficiency virus infection. The Syphilis and HIV Study Group. N Engl J Med 1997;337:307-14.

3. Marra CM. Update on neurosyphilis. Curr Infect Dis Rep 2009; 11:127-34.

4. McVey M, Cameron W, MacPherson P. When infections collide: gummatous syphilis in an HIV-infected patient. Int J Infect Dis 2010;14(Suppl 3):e283-6.

5. Mitsonis $\mathrm{CH}$, Kazariou E, Dimopoulous N, et al. Incidence and classical presentation of neurosyphilis: a retrospective study of 81 cases. Int J Neurosci 2008;118:1251-7. 
6. Hotson JR. Modern neurosyphilis. A partially treated chronic meningitis. West J Med 1981;135:191-200.

7. Knudsen RP. Neurosyphilis: overview of syphilis of the CNS Medscape Reference 2011. Available: http://emedicine.medscape .com/article/1169231 (accessed 2012 Oct. 15).

8. Yao Y, Huang E, Xie B, et al. Neurosyphilis presenting with psychotic symptoms and status epilepticus. Neurol Sci 2012;33: 99-102.

9. Aldave AJ, King JA, Cunningham ET Jr. Ocular syphilis. Curr Opin Ophthalmol 2001;12:433-41.

10. Rompalo AM, Joesoef R, O'Donnell JA, et al. Clinical manifestations of early syphilis by HIV status and gender: results of the syphilis and HIV study. Sex Transm Dis 2001;28:158-65.

11. Mao S, Liu Z. Neurosyphilis manifesting as lightning pain. Eur J Dermatol 2009;19:504-6.

12. Timmermans M, Carr J. Neurosyphilis in the modern era. $\mathrm{J} \mathrm{Neu}$ rol Neurosurg Psychiatry 2004;75:1727-30.

13. Carr J. Parkinsonism secondary to neurosyphilis. Mov Disord 2009;24:1407.

14. Shah BB, Lang AE. Acquired neurosyphilis presenting as movement disorders. Mov Disord 2012;27:690-5.

15. Sinha S, Harish T, Taly AB, et al. Symptomatic seizures in neurosyphilis: an experience from a university hospital in south India. Seizure 2008;17:711-6.

16. Garber SJ, Christmas TJ, Rickards D. Voiding dysfunction due to neurosyphilis. Br J Urol 1990;66:19-21.

17. Kinghorn GR. Infectious diseases. In: Cohen J, Powderly WG editors. Syphilis. 2nd ed. St Louis (MO): Mosby; 2004. p. 807-16.

18. Zetola NM, Klausner JD. Syphilis and HIV: an update. Clin Infect Dis 2007;44:1222-8.

19. Marra CM, Maxwell CL, Smith SL, et al. Cerebrospinal fluid abnormalities in patients with syphilis: association with clinical and laboratory features. J Infect Dis 2004;189:369-76.

20. Zifko U, Wimberger D, Lindner K, et al. MRI in patients with general paresis. Neuroradiology 1996;38:120-3.

21. Winston A, Marriot D, Brew B. Early syphilis presenting as a painful polyradiculopathy in an HIV positive individual. Sex Transm Infect 2005;81:133-4.

22. Kyebambe PS. Neurosyphilis masquerading as hemiparesis and
Jacksonian epilepsy in an HIV positive patient: a case report. Afr Health Sci 2010;10:211-4

23. Hajjaj I. Optic atrophy revealing neurosyphilis. Presse Med 2010;39:845-6

24. Ducas J, Robson HG. Cerebrospinal fluid levels during treatment for latent syphilis. JAMA 1981;246:2583-4.

25. Hook EW III, Martin DH, Stephens J, et al. A randomized, comparative pilot study of azithromycin versus benzathine penicillin G for treatment of early syphilis. Sex Transm Dis 2002;29:486-90.

Affiliations: From the Department of Medicine (Costiniuk, MacPherson), Division of Infectious Diseases, The Ottawa Hospital; the Department of Biochemistry, Microbiology and Immunology (Costiniuk, MacPherson), Faculty of Medicine, University of Ottawa; and the Ottawa Hospital Research Institute (MacPherson), Ottawa, Ont.

Contributors: Both authors contributed equally to the writing and revision of the manuscript and approved the final version submitted for publication.

Acknowledgements: The authors gratefully acknowledge Drs. Ruxandra Antochi, Douglas Green, Vivek Patel, Mukul Sharma and Claire Touchie for contributing to the care of these patients and for thoughtful review of the manuscript.

\section{Resources for physicians}

- Public Health Agency of Canada: Canadian Guidelines on Sexually Transmitted Infections (www.phac-aspc.gc.ca/std-mts/sti-its)

- Toronto Public Health: Syphilis Laboratory Interpretation (www.toronto.ca /health/professionals/communicable_diseases/pdf/syphilis_lab_interpretation .pdf) - a summary chart to assist in the interpretation of results of laboratory tests for syphilis and a summary of treatment recommendations

\section{Change of address}

We require 6 to 8 weeks' notice to ensure uninterrupted service. Please send your current mailing label, new address and the effective date of change to:

\section{CMA Member Service Centre}

1870 Alta Vista Dr., Ottawa ON K1G 6R7

tel $888855-2555$ or $613731-8610 \times 2307$

fax 613 236-8864

cmamsc@cma.ca

\section{Changement d'adresse}

II nous faut de 6 à 8 semaines d'avis afin de vous assurer une livraison ininterrompue. Veuillez faire parvenir votre étiquette d'adresse actuelle, votre nouvelle adresse et la date de la prise d'effet du changement, à l'attention du

Centre des services aux membres de l'AMC 1870, prom. Alta Vista, Ottawa ON K1G 6R7 tél 888 855-2555 ou $613731-8610$ ×2307 fax 613 236-8864 cmamsc@cma.ca

\section{ASSOCIATION MÉDICALE CANADIENNE

